\title{
INCIDENCIA DEL IMPUESTO A SOLCA EN LOS PRÉSTAMOS DE CONSUMO, PERIODO NOVIEMBRE, DICIEMBRE DE 2014 Y ENERO DE 2015
}

SOLCA TAX INCIDENCE ON CONSUMER LOANS, PERIOD NOVEMBER, DECEMBER 2014 AND JANUARY 2015

\section{MARÍA FERNANDA PELCHOR ZAMBRANO', JORGE ENRIQUE CALDERÓN SALAZAR², ELSA GENOVEVA MAYORGA ${ }^{3}{ }^{3}$}

1 Universidad de Especialidades Espíritu Santo, Guayaquil, Ecuador. mpelchor@uees.edu.ec

2 Universidad de Especialidades Espíritu Santo, Guayaquil, Ecuador. jecalder@uees.edu.ec

3 Universidad de Especialidades Espiritu Santo, Guayaquil, Ecuador. emayorga@uees.edu.ec

RESUMEN

El estado ecuatoriano el 27 de octubre de 2014 puso en vigencia un impuesto sobre los préstamos de consumo con la finalidad de financiar tanto en investigaciones como en tratamiento a los enfermos de cáncer que se atiendan en la Sociedad de Lucha contra el Cáncer SOLCA. Esta modalidad se extiende al uso de las tarjetas de crédito, es decir que por cada monto que se contrate el usuario debe pagar el $0.5 \%$ sobre dicho rubro; la incertidumbre se presenta en determinar si dicho aumento en el pago puede reprimir el uso de esta herramienta financiera, por el costo adicional que absorbe directamente el usuario. En esta investigación a través del uso de la elasticidad de la demanda se demostrará si la aplicación del impuesto afecta los préstamos de consumo.

PALABRAS CLAVE: impuestos, financiamiento, crédito, consumo, SOLCA, bancos privados.
The Ecuadorian government's October 27, 2014 put into effect a tax on consumer loans in order to fund both research and treatment of cancer patients who attend the Society to Combat Cancer SOLCA. This mode extends the use of credit cards, meaning that for every amount the user is contracted to pay $0.5 \%$ on those items; uncertainty arises in determining whether such an increase in payment can suppress the use of this financial tool, the additional cost directly absorbed by the user. It also seeks to demonstrate through the application of the elasticity of demand when the Ecuadorian population, which is more conservative alternative to buy goods with the raid on consumer, loans alternatives.

KEYWORDS: taxes, financing, credit, consumption, SOLCA, private banks. 
INTRODUCCIÓN

Los gobiernos crean impuestos para ofrecer una mejor calidad de vida a sus ciudadanos, a través de partidas presupuestarias que garanticen a todos los habitantes que están dentro de su jurisdicción, tengan acceso a la salud, la seguridad y la alimentación, los cuales son financiados a partir del ingreso de empresas o personas naturales que desempeñan una determinada actividad dentro del territorio nacional (Douglas \& Finnerty, 2002). Según Brown (2009) la creación de estos rubros debe definirse en función a la oportunidad que ofrece cada segmento de mercado y que ello no perjudique la economía de cada familia, mostrando al Estado como un aliado principal del desarrollo social en una comunidad.

Para que una economía se desarrolle, necesita la presencia del consumo que facilite el intercambio de necesidades y traspaso del dinero como instrumento de transacción (Cervantes, 2002), y que en cada operación se genere impuestos que financien al gobierno para que proponga mejoras a los sectores más vulnerables de la población (González \& Rodríguez, 2006), pero el escenario en análisis se centra en estudiar un impuesto que se genera por la adquisición del dinero para el consumo; es decir, un rubro que podría no generarse si la población ecuatoriana no consume, poniendo en riesgos otros ingresos para el Estado como el Impuesto al Valor Agregado (IVA) e Impuesto a los Consumos Especial (ICE) (Calderón, 2002).

El BCE (2014) afirma que: "el 62\% del volumen de crédito de consumo con tarjetas de crédito está concentrado en 6 instituciones financieras en el Ecuador: Diners Club (15,88\%), Banco Pichincha (14,73\%), Banco de Guayaquil (12,15\%), Solidario (7,40\%), Cooperativa JEP $(7,40 \%)$ y Pacificard $(4,68 \%)$ " (p.2). El escenario decretado por el Gobierno Ecuatoriano consiste en que cada transacción que utilice préstamos de consumos se le sumará adicionalmente el impuesto de contribución a SOLCA del $0.5 \%$ dicho rubro se paga de forma anual al Estado Ecuatoriano, cuya vigencia entró a partir del 27 de octubre de 2014, quedando fuera de este recaudo y cobro a clientes que tengan préstamos de consumo en aquellas instituciones reguladas por la Superintendencia de Economía Popular y Solidaria (SEPS) (El Universo, 2014).

Al final de este estudio se busca determinar si en los meses de noviembre y diciembre de 2014 así como también enero de 2015 la demanda de préstamos de consumo disminuyó o aumentó.
REVISIÓN DE LA LITERATURA

El artículo de investigación abarca temas relacionados con los impuestos y la forma de recaudación hacia la ciudadanía en general con el objetivo de determinar si la incursión del nuevo rubro del impuesto a SOLCA incide directamente o no en la demanda de préstamos de consumo. De acuerdo a MSP (2013) la tasa de mortalidad por causa de cáncer es de 64 personas por cada 100.000 habitantes, donde los tumores malignos representan el $80 \%$ de esta causa, seguida por el cáncer de estómago, sistema hematopoyético, tiroides, colorecto, hígado, ganglios linfáticos, pulmón, y cuello de útero, siendo la población más expuesta aquellas mayores a los 65 años de edad.

Esta problemática motiva a buscar una fuente de financiamiento que promueva la investigación de prevenir el cáncer en el Ecuador, y que ello ayude a disminuir la tasa de mortalidad expuesta en el portal del Ministerio de Salud Pública (El Universo, 2014); el gobierno de turno a través de la Asamblea Nacional aprobó la ley de contribuir con el $0.5 \%$ en todo tipo de créditos de consumo de aquella población que ha obtenido de entidades financieras privadas y controladas por la Superintendencia de Bancos, todo esto fijado en la resolución No. 003-2014-F por la Junta de Política y Regulación Monetaria y Financiera.

Larraín y Sachs (2010) indican que los impuestos son herramientas políticas generadores de recursos financieros para adquirir los bienes públicos. De igual manera, tratan de redistribuir la riqueza de los habitantes de un determinado país, permitiéndoles gozar en iguales condiciones temas de salud, vivienda y alimentación, también indica que en sentido general los gobiernos utilizan cinco tipos de impuestos: impuestos sobre la renta, impuestos para la seguridad social, impuestos sobre las ventas, impuestos a las propiedades, impuestos al consumo (Plus, 2007).

Al respecto Phillip (2011) señala que un impuesto sobre el consumo es un impuesto gravado como una cantidad específica por unidad de producto. También se denomina impuesto específico y su impacto depende de la elasticidad de la demanda del segmento de mercado (Bigne \& Cuenca, 2010). En esta investigación la demanda lo componen aquellos ecuatorianos que son sujetos de crédito y tienen bajo sus obligaciones financieras el pago o posesión de un instrumento que les permita contratar préstamos de consumo (Fernández, 2007). Por el lado de la oferta la componen el número de entidades financieras clasificadas por Superintendencia 
de Bancos y Seguros (2015) como bancos privados sin tomar en consideración la clasificación que éste tenga en el sistema financiero ecuatoriano.

\section{IMPUESTO SOBRE EL CONSUMO}

Con relación a Parkin (2007) un impuesto sobre el ingreso de un trabajo, debilita el incentivo a trabajar, si relacionamos este concepto a la herramienta financiera de la tarjeta de crédito, un impuesto causa un efecto negativo al ecuatoriano de usar el mismo para sus compras. Según Luchena (2007) al momento de ser reemplazado el dinero por una herramienta financiera como el préstamo de consumo a través de una tarjeta de crédito, y ayudado por un medio electrónico que transacción con el banco emisor, el usuario no contempla la salida de dinero, y ello contribuye a generar el endeudamiento.

La aplicación de un impuesto sobre un escenario que el usuario no se siente con restricción de límites para el consumo independiente de su cupo de crédito, garantiza que las probabilidades de recaudación del mismo se incrementen (Lawrence, 2010). Por lo tanto la recaudación de impuesto a través del consumo es una herramienta efectiva tanto como el impuesto al valor agregado, como el impuesto a la renta, ya que el cliente además de sentirse tentado por el artículo, no tiene que usar dinero para adquirirlo, ni alterar su economía en recurso financiero en ese momento (Navarro, 2003).

\section{METODOLOGÍA}

El presente diseño de la investigación tiene la aplicación del método explicativo con datos cuantitativos, ya que busca determinar el efecto de aplicar un impuesto directamente al monto de consumo de los préstamos de los ecuatorianos, y una investigación descriptiva con datos cuantitativos sobre las reacciones de la cartera de los clientes que posee la institución de bancos privados. Los datos obtenidos fueron en base a los reportes que genera el Banco Central del Ecuador sobre la evolución del sistema financiero, tanto en número de créditos otorgados como el monto de los desembolsos de cada cliente.

El sistema de crédito bancario ecuatoriano, para elegir su población involucra a sus solicitantes a un proceso de evaluación financiera a fin de que se tenga la seguridad que el individuo sea sujeto de crédito y que tenga los recursos financieros y económicos para cubrir dicha obligación, por lo tanto este número es variable ya que un mismo individuo puede solicitar distintas operaciones y sobre todo si el mismo tiene en su poder una tarjeta de crédito (Superintendencia de Bancos y Seguros del Ecuador, 2015).

La población se basa en los datos proporcionados por los informes del Banco Central del Ecuador, de tipo real y sin la necesidad de un tipo de estimación para hallar la totalidad de la población. Al tratar datos reales y totales se suprime el cálculo de la muestra y es una ventaja para la investigación porque se evalúa un comportamiento real de la situación que se analiza (Hurtado, 2000).

Para establecer una conclusión del impuesto a SOLCA sobre los préstamos de consumo, se utilizó el criterio de la elasticidad de la demanda, el cual mide el cambio de la cantidad consumida en un mercado por algún cambio en el precio. A continuación se exponen datos históricos sobre el nivel de préstamos de consumo concedidos por las instituciones financieras entre noviembre y diciembre del año 2013 y enero de 2014 a fin de establecer criterios de sensibilidad sobre la oportuna incorporación de un impuesto a este tipo de créditos que actualmente está en vigencia y compararlos con el período entre noviembre y diciembre del año 2014 y enero de 2015 con la aplicación del impuesto.

ANÁLISIS DE RESULTADOS

A continuación se muestra la evolución del sistema de crédito en el sistema financiero privado entre noviembre de 2013 y noviembre de 2014 así como un comparativo con octubre de 2014.

TABLA 1. VOLUMEN DE CRÉDITO OTORGADO POR LOS BANCOS PRIVADOS MES DE NOVIEMBRE 2014

\begin{tabular}{|c|c|c|c|c|c|}
\hline \multirow[b]{2}{*}{$\begin{array}{l}\text { VOLÚMEN DE } \\
\text { CRÉDITO POR } \\
\text { SEGMENTO }\end{array}$} & \multicolumn{3}{|c|}{ MILLONES USD } & \multicolumn{2}{|c|}{ VARIACIÓN MENSUAL } \\
\hline & NOV-13 & OCT-14 & NOV-14 & $\begin{array}{l}\text { OCT-14 A } \\
\text { NOV-14 }\end{array}$ & $\begin{array}{l}\text { NOV-13 A } \\
\text { NOV-14 }\end{array}$ \\
\hline A,C, Simple & 54,4 & 59,8 & 61,3 & $2,51 \%$ & $12,68 \%$ \\
\hline A,C, Ampliada & 27,5 & 27 & 24,7 & $-8,52 \%$ & $11 \%$ \\
\hline Minorista & 46,1 & 45 & 51,1 & $13,56 \%$ & $-3,56 \%$ \\
\hline PYMES & 191,1 & 206,6 & 184,3 & $-11 \%$ & $-3,56 \%$ \\
\hline Consumo & 277,2 & 310,6 & 268,7 & $-13,46 \%$ & $-3,07 \%$ \\
\hline Vivienda & 40,3 & 39,7 & 37,6 & $-5,29 \%$ & $-6,70 \%$ \\
\hline Empresarial & 186,1 & 214,2 & 165,8 & $-22,60 \%$ & $-10,91 \%$ \\
\hline Corporativo & 919,7 & 950,2 & 797,1 & $-16,11 \%$ & $-13,33 \%$ \\
\hline $\begin{array}{l}\text { Total volumen de } \\
\text { crédito }\end{array}$ & 1742,4 & 1853 & 1590,6 & $-7,59 \%$ & $-3,03 \%$ \\
\hline $\begin{array}{l}\text { Total número de } \\
\text { operaciones }\end{array}$ & 368,149 & 399,887 & 384,613 & $-3,82 \%$ & $4,47 \%$ \\
\hline
\end{tabular}

Fuente: Publicaciones mensuales económicas. Noviembre 2014.

Tomado de: Banco Central del Ecuador, 2014. 
En la tabla 1 se visualiza que el volumen de crédito en noviembre de 2014 por parte de los bancos privados, tuvo una leve disminución del $3.07 \%$ entre años 2013 y 2014, y $13.46 \%$ entre los meses de octubre y noviembre, lo que se deduce que en el mes de noviembre los usuarios estuvieron más cautelosos en su comportamiento de compra. En este escenario los consumidores ya están contemplado el pago del $0.5 \%$ sobre la base de cualquier préstamo de consumo, recaudando a favor de SOLCA 1.34 millones de dólares, dicho valor es cargado en el estado de cuenta de los titulares de tarjetas de crédito y préstamos de consumo.

TABLA 2. VOLUMEN DE CRÉdITO OTORGAdO POR LOS BANCOS PRIVADOS MES DE DICIEMBRE 2014

\begin{tabular}{|c|c|c|c|c|c|}
\hline \multirow[b]{2}{*}{$\begin{array}{l}\text { VOLÚMEN DE } \\
\text { CRÉDITO POR } \\
\text { SEGMENTO }\end{array}$} & \multicolumn{3}{|c|}{ MILLONES USD } & \multicolumn{2}{|c|}{ VARIACIÓN MENSUAL } \\
\hline & DIC-13 & NOV-14 & DIC-14 & $\begin{array}{l}\text { DIC-13 A } \\
\text { DIC14 }\end{array}$ & $\begin{array}{l}\text { NOV-14 A } \\
\text { DIC-14 }\end{array}$ \\
\hline$A, C$, Simple & 41,8 & 61,3 & 45,2 & $-26,26, \%$ & $8,13 \%$ \\
\hline A,C, Ampliada & 21,4 & 24,7 & 19,1 & $-22,67, \%$ & $-10,75 \%$ \\
\hline Minorista & 40,7 & 51,1 & 44,4 & $-13,11, \%$ & $9,09 \%$ \\
\hline PYMES & 184,6 & 184,3 & 190,3 & $3,26, \%$ & $3,09 \%$ \\
\hline Consumo & 256,8 & 268,7 & 282,3 & $5,06, \%$ & $9,93 \%$ \\
\hline Vivienda & 34,8 & 37,6 & 40,6 & $7,98, \%$ & $16,67 \%$ \\
\hline Empresarial & 210,2 & 165,8 & 188,2 & $13,51, \%$ & $-10,47 \%$ \\
\hline Corporativo & 942,6 & 797,1 & 928,0 & $16,42, \%$ & $-1,55 \%$ \\
\hline $\begin{array}{l}\text { Total volumen de } \\
\text { crédito }\end{array}$ & 1732,9 & 1590,6 & 1738,1 & $-1,98, \%$ & $3,02 \%$ \\
\hline $\begin{array}{l}\text { Total número de } \\
\text { operaciones }\end{array}$ & 419,948 & 384,613 & 420,360 & $9,29, \%$ & $0,10 \%$ \\
\hline
\end{tabular}

Fuente: Publicaciones mensuales económicas. Diciembre 2014.

Tomado de: Banco Central del Ecuador, 2014.

En la tabla 2 se visualiza el comportamiento del volumen de crédito otorgado por los bancos privados en el mes de diciembre, esta época se caracteriza por la entrega de regalos producto de las fiestas navideñas; se observa que el consumo aumentó en un $9.93 \%$ con relación a diciembre de 2013 y $5.06 \%$ con su mes inmediato anterior de noviembre de 2014 sin embargo su aumento aún no compensa la caída sufrida del $13.46 \%$ mostrada en la tabla 1 , lo que significa que los consumidores aún tienen cierto malestar de cargar a sus cuentas por cobrar valores adicionales tanto en tasas como en impuestos.
TABLA 3. VOLUMEN DE CRÉDITO OTORGADO POR LOS BANCOS PRIVADOS MES DE ENERO 2015

\begin{tabular}{|c|c|c|c|c|c|}
\hline \multirow[b]{2}{*}{$\begin{array}{l}\text { VOLÚMEN DE } \\
\text { CRÉDITO POR } \\
\text { SEGMENTO }\end{array}$} & \multicolumn{3}{|c|}{ MILLONES USD } & \multicolumn{2}{|c|}{ VARIACIÓN MENSUAL } \\
\hline & ENE-14 & DIC-14 & ENE-15 & $\begin{array}{l}\text { DIC-14 A } \\
\text { ENE-15 }\end{array}$ & $\begin{array}{l}\text { ENE-14 A } \\
\text { ENE15 }\end{array}$ \\
\hline A,C, Simple & 52,4 & 45,2 & 42,8 & $-5,31, \%$ & $-18,32 \%$ \\
\hline A,C, Ampliada & 23,1 & 19,1 & 17,1 & $-10,47, \%$ & $-25,97 \%$ \\
\hline Minorista & 38,9 & 44,4 & 30,4 & $-177,41, \%$ & $-21,85 \%$ \\
\hline PYMES & 139,9 & 190,3 & 134,5 & $-29,32, \%$ & $-3,86 \%$ \\
\hline Consumo & 245,2 & 282,3 & 235,0 & $-16,76, \%$ & $-4,16 \%$ \\
\hline Vivienda & 28,7 & 40,6 & 32,2 & $-20,69, \%$ & $12,20 \%$ \\
\hline Empresarial & 162,3 & 188,2 & 154,5 & $-17,91, \%$ & $-4,81 \%$ \\
\hline Corporativo & 731,3 & 928 & 792,3 & $-14,62, \%$ & $8,34 \%$ \\
\hline $\begin{array}{l}\text { Total volumen de } \\
\text { crédito }\end{array}$ & 1421,8 & 1738,1 & 1438,8 & $-18,33, \%$ & $-7,30 \%$ \\
\hline $\begin{array}{l}\text { Total número de } \\
\text { operaciones }\end{array}$ & 336,133 & 420,36 & 01,602 & $-28,25, \%$ & $-10,27 \%$ \\
\hline
\end{tabular}

Fuente: Publicaciones mensuales económicas. Enero 2015.

Tomado de: Banco Central del Ecuador, 2014.

En la tabla 3 se muestra el reporte del total de créditos en monto que han desembolsado los bancos privados, en él se detalla que el préstamo de consumo generó un total de \$235 millones de dólares, un valor por debajo del $4.16 \%$ con relación al año anterior y $16.76 \%$ con respecto a diciembre del año 2014. Esto sigue aportando a mantener una tendencia baja en cuanto a la solicitud de préstamos de esta índole que no se recupera desde la partida del mes de octubre de 2014 cuando se comenzó con el análisis.

Sin embargo a pesar de que el impuesto a SOLCA no contempla un impacto en desembolsos de dinero en préstamos que se destinan para otras actividades, el mes de enero ha representado una baja en todos los sectores, debido a que la demanda de artículos y producción bajó luego de las festividades navideñas.

La demanda en créditos no solo se vio afectada por el impuesto al consumo sino por el endeudamiento adquirido por la población en el mes de diciembre. Sin embargo con relación a enero del año anterior no se generó en igual o mayor proporción, teniendo en cuenta que la economía debe crecer todos los años y más aún en la ecuatoriana donde las personas tienen la facilidad de aplicar una tarjeta de crédito o ser sujetos de un préstamo bancario a corto y largo plazo sin mayor restricción. 


\section{Comportamiento de préstamos de}

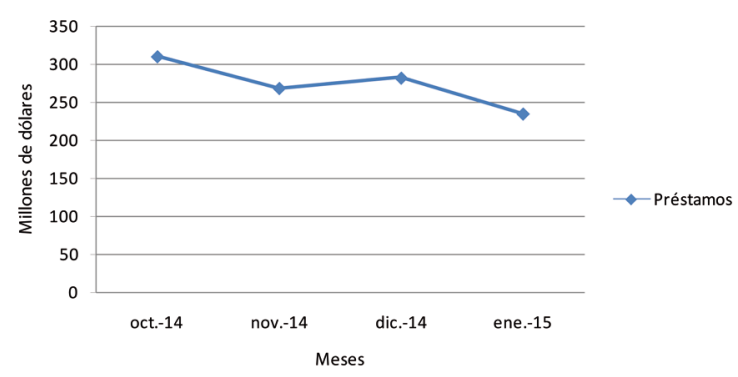

Figura 1. Comportamiento de préstamos de consumo Fuente: Publicaciones mensuales económicas.

En el figura 1 se muestra cómo ha sido el comportamiento del préstamo de consumo desde que se implementó el impuesto a SOLCA, con un horizonte de análisis entre los meses de octubre, noviembre, diciembre de 2014 y enero de 2015. En el eje de las ordenadas se detallan los dólares que se desembolsaron los meses en análisis y en el eje de las abscisas el tiempo que se recogió la información.

El primer mes que se obtuvo información fue en octubre de 2014 donde el crédito de consumo desembolso en totalidad de los bancos privados 310.50 millones dólares, este punto fue el más alto de toda la investigación, ya que en los meses siguientes la tendencia fue negativa ya que no se superó esta cifra. En este mes recién se puso en vigencia el Impuesto a SOLCA por lo tanto aquí los consumidores recibieron en primer lugar el impacto de este rubro.

En el mes de noviembre, el escenario fue poco alentador ya que el total de desembolso cerró en \$268.7 millones de dólares, aproximadamente $\$ 40$ millones por debajo de lo que generó octubre de 2014, la deducción obtenida fue que los consumidores se muestran represivos al gasto frente al acercamiento de una época navideña que genera más compras a las familias ecuatorianas, por lo tanto se esperaba que en el mes de diciembre el comportamiento suba y se obtenga un desembolso mayor que todos los meses en análisis.

En el mes de diciembre el préstamo de consumo desembolsó \$282.3 millones de dólares, es decir \$13.60 millones de dólares más que en noviembre pero $\$ 28.2$ menos millones de dólares que en el mes de octubre, aquí se evidencia que las personas están siendo más cautelosas con este tipo de préstamos, ya que en este mes debía aumentar considerablemente el consumo por las promociones navideñas que ofertan los diferentes almacenes de las ciudades.
En el mes de enero, este producto financiero desciende con un cierre en $\$ 235$ millones de dólares en desembolsos es decir $\$ 47.30$ millones de dólares menos entregados a la población con respecto a diciembre de 2014 y $\$ 33.70$ millones de dólares con relación a noviembre de 2014, luego de pasar por una serie de ofertas en el mes anterior.

Más adelante se hace una comparativa de los meses en análisis pero de diferentes años con la finalidad de que a manera visual se pueda establecer premisas de un escenario sin la intervención del impuesto a SOLCA.

\section{Comparación del consumo entre los años}

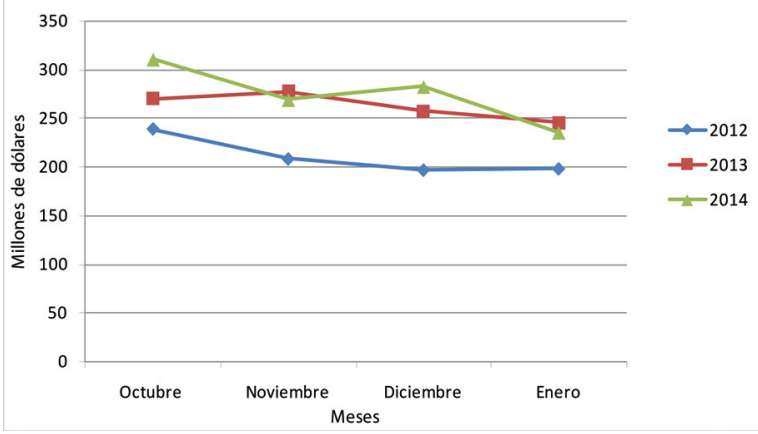

Figura 2. Comportamiento del préstamo de consumo desembolsado entre los años 2012, 2013 y 2014

Fuente: Publicaciones mensuales económicas.

En el figura 2 se observa que el mes de octubre es el tiempo en que las personas acuden a una entidad bancaria privada con mayor frecuencia a excepción del año 2013 donde el mes de noviembre se impuso con 7 millones de dólares desembolsados adicionalmente. Se muestra también que el comportamiento entre estos meses es negativo, sin embargo este producto financiero creció de manera considerable entre los años 2012 y 2013 debido a la brecha mostrada en la figura donde la línea de recreación no se intercepta en ningún mes.

En el año 2014 este panorama cambia totalmente ya que se observa como el comportamiento intercepta los meses de noviembre, diciembre y enero del año anterior, terminando además en un monto desembolsado inferior en enero de 2014, por lo tanto con esta evidencia se evidencia que de manera directa el nuevo impuesto está incidiendo considerablemente en la forma de adquirir préstamos para el consumo y que el escenario en los próximos periodos llevará a que la población se resista a contratarlos y busque otras maneras de apalancarse financieramente. 
Esto limita a los bancos privados y representa una desventaja competitiva entre las cooperativas de ahorro y crédito, ya que sus montos tienen menos conceptos adicionales de pagar a diferencia de la tasa de interés, pero representa una alternativa de mayor ahorro frente al producto financiero de los bancos privados. Otro concepto que afecta es a las tarjetas de crédito, ya que al final de su corte generan notas de débito adicionales al titular llevando a reducir su cupo de crédito e impedirlo de realizar mayores compras en otros establecimientos comerciales. En el análisis de resultados se muestra a través del criterio de la elasticidad de la demanda la relación existente entre el precio y cantidad mostrados por los desembolsos de préstamos de consumos expedidos por el Banco Central del Ecuador. Se realizan dos tipos de análisis:

El primero comprende un comparativo entre meses del mismo año a fin de establecer si la adición del valor del Impuesto a SOLCA en los estados de cuenta de los consumidores va afectando o no directamente en el uso del producto financiero, o a su vez no representa ninguna restricción en seguir usando este medio como forma de pago. Sin embargo el primer análisis esta susceptible a una desigualdad de motivaciones al consumo ya que en los meses contemplados no todos muestran el mismo ritmo en que las casas comerciales o prestadoras de servicios motivan al público a adquirir sus artículos. Un ejemplo radica que en diciembre el consumo aumenta debido a las promociones navideñas que realizan los centros comerciales a diferencia de octubre y noviembre donde estos ofertantes recién se abarrotan de productos para ofertarlos en diciembre, por otro lado en enero el consumo tiende a bajar ya que los consumidores destinaron sus recursos financieros en diciembre y en este mes comienzan a pagar sus deudas.

Por lo tanto en respuesta a esta desigualdad se realiza otra comparación que comprende el mes en análisis vs el corriente del año anterior, con esto se obtiene que si en el mes de diciembre se realice ofertas por navidad se obtenga el mismo resultado en créditos o mayor de ser el caso contra el año pasado, si existe una baja se pueda concluir que otro factor está ocasionando una disminución o restricción en los consumidores por al utilizar este producto financiero como herramienta para realizar sus compras.

El primer parámetro a analizar es el comportamiento de la demanda entre el mes de octubre y noviembre de 2014, con la variable indepen- diente determinada en el precio por adquisición del crédito y la variable dependiente el número de operaciones llevadas por los bancos.

\section{ELASTICIDAD DE DEMANDA ENTRE OCTUBRE Y NOVIEMBRE DE 2014}

Por su parte el BCE (2014) indica que el comportamiento de créditos de consumo estuvo en \$310.50 millones de dólares a octubre de 2014 con una participación de Bancos del 80.93\% en ese segmento de mercado.

TABLA 4. OPERACIONES Y VOLUMEN DE CRÉDITO ENTRE OCTUBRE Y NOVIEMBRE DE 2014

\begin{tabular}{lllll}
\hline AÑO & MES & $\begin{array}{l}\text { NÚMERO DE } \\
\text { OPERACIONES }\end{array}$ & \multicolumn{2}{l}{$\begin{array}{l}\text { VOLUMEN DE CRÉDITOS DE } \\
\text { CONSUMO }\end{array}$} \\
\hline 2014 & octubre & 399,887 & $\$ 310.50$ \\
2014 & noviembre & 384,613 & $\$ 268.70$ \\
\hline
\end{tabular}

Fuente: Publicaciones mensuales económicas.

Tomado de: Banco Central del Ecuador.

En la tabla 4 se muestra el número de operaciones surgidas entre octubre y noviembre del año 2014 seguida por el volumen de crédito de consumo otorgado por los bancos del sector privado, estos datos sirven para determinar la elasticidad de la demanda, y si la aplicación del impuesto a SOLCA incide de manera considerable para afectar las solicitudes de préstamos en calidad de consumo en el Ecuador.

La elasticidad que se obtiene es de 0.26 , considerada una demanda inelástica, por lo cual se deduce que de octubre a noviembre del año 2014 la aplicación del impuesto a SOLCA sobre préstamos de consumo no ha afectado de manera considerable la demanda de créditos por parte de los ecuatorianos en el sector de bancos privados.

\section{ELASTICIDAD DE DEMANDA \\ ENTRE NOVIEMBRE DE 2013 Y NOVIEMBRE DE 2014}

Otro comparativo a realizar es el comportamiento de la demanda del mismo mes pero en diferentes años sobre los requerimientos de créditos de consumo, ya que de ese modo se mide en iguales variables, ya sea en condiciones de requerimiento de capital, nivel de publicidad en motivación al consumismo, y poder adquisitivo de los ecuatorianos en aquellas épocas.

De igual manera el BCE (2013) en su boletín de evolución de crédito muestra que en noviembre de 2013 las operaciones crediticias ascendieron a 368.149 transacciones desembolsando un total de \$277.20 millones de dólares. 
TABLA 5. OPERACIONES Y VOLUMEN DE CRÉDITO ENTRE NOVIEMBRE DE 2013 Y NOVIEMBRE DE 2014

\begin{tabular}{|lllll|}
\hline AÑO & MES & $\begin{array}{l}\text { NÚMERO DE } \\
\text { OPERACIONES }\end{array}$ & $\begin{array}{l}\text { VOLUMEN DE CRÉDITOS DE } \\
\text { CONSUMO }\end{array}$ & \\
\hline 2013 & noviembre & 368,149 & & $\$ 277.20$ \\
2014 & noviembre & 384,613 & $\$ 268.70$ \\
\hline
\end{tabular}

Fuente: Publicaciones mensuales económicas.

Tomado de: Banco Central del Ecuador.

En la tabla 5 se observa los datos obtenidos sobre los desembolsos realizados por los bancos del sector financiero privado donde claramente se muestra un descenso tanto en el número de operaciones como el número de créditos de consumo otorgados, estos valores al igual que la comparación anterior se proceden a tomar para conocer la elasticidad de la demanda, y su impacto en la forma de solicitar dichos créditos. El resultado que se obtiene de la aplicación de la fórmula es de 1.40 siendo una demanda elástica, lo que se deduce que los consumidores están usando este financiamiento para casos de emergencia y compras de bajo nivel de endeudamiento, ya que disminuyó su nivel de adquirir préstamos.

ELASTICIDAD DE DEMANDA

ENTRE NOVIEMBRE Y DICIEMBRE DE 2014

Igualmente el BCE (2014) indica que el comportamiento de créditos de consumo estuvo en $\$ 268.70$ millones de dólares a noviembre de 2014.

TABLA 6. OPERACIONES Y VOLUMEN DE CRÉDITO ENTRE NOVIEMBRE Y DICIEMBRE DE 2014

\begin{tabular}{lllll}
\hline AÑO & MES & $\begin{array}{l}\text { NÚMERO DE } \\
\text { OPERACIONES }\end{array}$ & $\begin{array}{l}\text { VOLUMEN DE CRÉDITOS DE } \\
\text { CONSUMO }\end{array}$ & \\
\hline 2014 & noviembre & 384,613 & & $\$ 268.70$ \\
2014 & diciembre & 420,360 & $\$ 282.30$ \\
\hline
\end{tabular}

Fuente: Publicaciones mensuales económicas.

Tomado de: Banco Central del Ecuador.

En la tabla 6 se muestra el número de operaciones surgidas entre noviembre y diciembre del año 2014 seguida por el volumen de crédito de consumo otorgado por los bancos del sector privado, estos datos sirven para determinar la elasticidad de la demanda, y si la aplicación del impuesto a SOLCA incide de manera considerable para afectar las solicitudes de préstamos en calidad de consumo en el Ecuador.
La elasticidad que se obtiene es de 1.79 sien- $^{-}$ do una demanda elástica, por lo cual se deduce que de noviembre a diciembre del año 2014 la aplicación del impuesto a SOLCA sobre préstamos de consumo ha afectado de manera considerable la demanda de créditos por parte de los ecuatorianos en el sector de bancos privados, obligándolos a ser más cautelosos antes de usar este método como fuente de financiamiento a sus compras.

\section{ELASTICIDAD DE DEMANDA}

ENTRE DICIEMBRE DE 2013 Y DICIEMBRE DE 2014

Otro comparativo a realizar es el comportamiento de la demanda del mismo mes pero en diferentes años sobre los requerimientos de créditos de consumo, ya que de ese modo se mide en iguales variables, ya sea en condiciones de requerimiento de capital, nivel de publicidad en motivación al consumismo, y poder adquisitivo de los ecuatorianos en aquellas épocas. Para el BCE (2013) en su boletín de evolución de crédito, en diciembre de 2013 las operaciones crediticias ascendieron a 419.948 transacciones desembolsando un total de \$256.80 millones de dólares.

\begin{tabular}{|c|c|c|c|}
\hline & MES & $\begin{array}{l}\text { NÚMERO DE } \\
\text { OPERACIONES }\end{array}$ & $\begin{array}{l}\text { VOLUMEN DE CRÉDITOS DE } \\
\text { CONSUMO }\end{array}$ \\
\hline 2013 & diciembre & 419,948 & $\$ 256.80$ \\
\hline 2014 & diciembre & 420,360 & $\$ 282.30$ \\
\hline
\end{tabular}

Fuente: Publicaciones mensuales económicas.

Tomado de: Banco Central del Ecuador.

En la tabla 7 se observa los datos obtenidos sobre los desembolsos realizados por los bancos del sector financiero privado donde claramente se muestra un descenso tanto en el número de operaciones como el número de créditos de consumo otorgados, estos valores al igual que la comparación anterior se proceden a tomar para conocer la elasticidad de la demanda, y su impacto en la forma de solicitar dichos créditos. La elasticidad que se obtiene es de 0.01 , es decir una demanda perfectamente inelástica con ello se deduce que un cambio en el aumento del costo del dinero en préstamos de consumo no afecta en nada al número de operaciones que se soliciten en los bancos privados. Este efecto se proyecta en base a las festividades navideñas de la época donde el consumidor se ve abaratado por promociones y descuentos que facilitan la adquisición de bienes y servicios. 
ELASTICIDAD DE DEMANDA

ENTRE DICIEMBRE DE 2014 Y ENERO DE 2015

De acuerdo al BCE (2015) en el mes de enero el comportamiento de créditos de consumo obtuvo un monto total de operaciones de \$235 millones de dólares. Posteriormente se procede a realizar un comparativo con el mes inmediato anterior a fin de establecer la sensibilidad de mercado luego de pasar las festividades y tener la influencia del impuesto a SOLCA en los consumidores.

TABLA 8. OPERACIONES Y VOLUMEN DE CRÉDITO ENTRE DICIEMBRE DE 2014 Y ENERO DE 2015

\begin{tabular}{lllrl}
\hline AÑO & MES & $\begin{array}{l}\text { NÚMERO DE } \\
\text { OPERACIONES }\end{array}$ & $\begin{array}{l}\text { VOLUMEN DE CRÉDITOS DE } \\
\text { CONSUMO }\end{array}$ & \\
\hline 2014 & diciembre & 420,36 & $\$ 282.30$ \\
2015 & enero & 301,602 & $\$ 235.00$ \\
\hline
\end{tabular}

Fuente: Publicaciones mensuales económicas.

Tomado de: Banco Central del Ecuador.

En la tabla 8 se muestra los resultados obtenidos en desembolsos y número de operaciones entre diciembre de 2014 y enero de 2015 proporcionados por el Banco Central del Ecuador. En cuanto al mes de diciembre se evidencia una baja tanto en operaciones como en créditos, esto es un comportamiento esperado debido a que en este mes los almacenes y las tiendas no cuentan con ofertas y combos para la venta, con los cual el consumidor se siente un poco desmotivado en adquirirlos y más restringido por el costo adicional que tiene que pagar al realizar un crédito de consumo.

La elasticidad que se obtiene es de 1.79, siendo elástica, por lo cual se deduce que de diciembre de 2014 a enero del año 2015 la aplicación del impuesto a SOLCA sobre préstamos de consumo ha generado un impacto considerable en la solicitud de este producto financiero conjuntamente con la baja de promociones por las casas comerciales y prestación de beneficios.

La variable de promociones navideñas conlleva a que el resultado del cálculo de la elasticidad sea susceptible a un criterio erróneo debido a que enero tiende hacer un mes de restricciones a compras ya que la población ecuatoriana sale de un mes de mayor endeudamiento como lo es diciembre, por lo tanto a continuación se realiza un análisis entre años del mismo mes a fin de conocer si la economía muestra algún cambio con la incorporación del nuevo impuesto.

\section{ELASTICIDAD DE DEMANDA \\ ENTRE ENERO DE 2014 Y ENERO DE 2015}

En este punto se analizan los resultados obtenidos en el mismo mes pero de diferentes años, con este análisis se comprueba bajo las mismas condiciones del mercado como el Impuesto a SOLCA genera aspectos positivos o negativos a las personas que solicitan el préstamo de consumo. Aquí se suprimen variables que incentivan la compra como lo ocurrido en diciembre, y se analizan meses en igual condiciones afectados por el endeudamiento masivo que provoca las festividades navideñas en el mes anterior.

El BCE (2015) en su boletín de evolución de crédito, en enero de 2014 las operaciones crediticias ascendieron a 336.133 transacciones desembolsando un total de \$245.20 millones de dólares en consumo, es decir mayor desembolso y operaciones que el mes de enero de 2015, los comparativos se muestran a continuación.

\begin{tabular}{|c|c|c|c|}
\hline AÑo & MES & NÚMERO DE OPERACIONES & $\begin{array}{l}\text { VOLUMEN DE CRÉDITOS DE } \\
\text { CONSUMO }\end{array}$ \\
\hline 2014 & enero & 336,133 & $\$ 245.20$ \\
\hline 2015 & enero & 301,602 & $\$ 235.00$ \\
\hline
\end{tabular}

Fuente: Publicaciones mensuales económicas.

Tomado de: Banco Central del Ecuador.

En la tabla 9 se evidencia que la economía no respondió de manera positiva en el año 2015, porque su solicitud en créditos fue más baja y los montos desembolsados fueron menores con relación al año 2014. Considerando que ambos meses han soportado las ofertas de diciembre, se concluye que existe un efecto negativo en el consumidor al adquirir estos tipos de préstamos por lo tanto se indica que el Impuesto a SOLCA en el mes de enero si se vio afectado y que es un producto financiero que al mantenerse en estas condiciones va a disminuir paulatinamente lo cual quita participación de mercado a los bancos privados.

La elasticidad que se obtiene es de 2.55, concluyendo que la demanda es elástica, por lo tanto el costo por el dinero prestado influido por el Impuesto a SOLCA, afecta la solicitud de créditos por este concepto. Con ello se restringe a la población a pensar dos veces antes de utilizar este producto financiero o en su caso a ser más selectivo en instituciones financieras al realizar un préstamo de consumo, ya que esta medida solo afecta a los bancos privados. 
DISCUSIÓN

Se evidencia que desde octubre a enero de 2015 la relación del precio con la cantidad demanda ha cambiado de ser inelástica a volverse totalmente elástica, debido a que el factor en Impuesto a SOLCA afecta directamente al monto que se le carga a los consumidores al momento de utilizar este medio de pago.

Este impuesto está desmotivando al consumo, y limitando el uso de la herramienta financiera, ya que directamente agrega una cantidad al monto adeudado perjudicando la economía de los consumidores. A continuación se muestra una gráfica resumiendo el análisis llevado a cabo de las elasticidades de la demanda entre meses del mismo año con una línea roja que representa el momento en que la demanda se volvió más sensible a los montos que debe adeudar a la entidad financiera, bajando el número de operaciones dadas entre los meses de octubre, noviembre, diciembre de 2014 y enero 2015.

A pesar de que este impuesto genera un rubro adicional de ingresos con relación al financiamiento de investigaciones contra lucha del cáncer afecta directamente al poder de compra de los consumidores y por ende a otros impuestos que se genera producto de estas transacciones como el ICE, IVA e Impuesto a la Salida de Divisas, ya que menor monto se disponga para comprar, menor cantidad de préstamos de estos conceptos el Estado ecuatoriano puede recaudar.

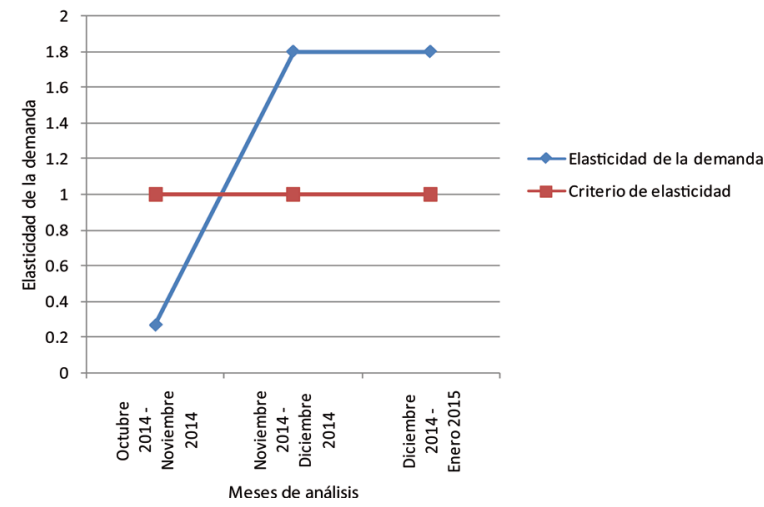

Figura 3. Evolución de la elasticidad de la demanda entre meses del mismo año

En los meses de noviembre a diciembre los consumidores mostraron una disminución en demanda al utilizar este tipo de financiamiento, llevando a que su contratación por deuda disminuya, y recurrir a otras fuentes de pago para adquirir bienes y servicios en el caso que continuara su mismo nivel de consumo. En los meses de diciembre a enero de 2015 el comportamiento se mantuvo constante, generando menos demanda en el monto de créditos y aumentando el costo del dinero, por ello se desembolsó menos recursos financieros. Esto avizora que en un futuro los bancos privados se sientan perjudicados de esta medida ya que aleja al consumidor y evita que los mismos tengan un desarrollo positivo en sus economías.

Con esto se deja en hipótesis si el Estado se ve afectado en relación a la recaudación de otros impuestos que percibe para el presupuesto general ya que si los consumidores cuentan con otras herramientas para financiar su calidad de vida, el efecto a la baja en recaudaciones no recibe un impacto considerable en impuestos, ni los consumidores ven disminuida su capacidad de compra, el único ofertante afectado serían las entidades financieras que deben retener dicho impuesto.

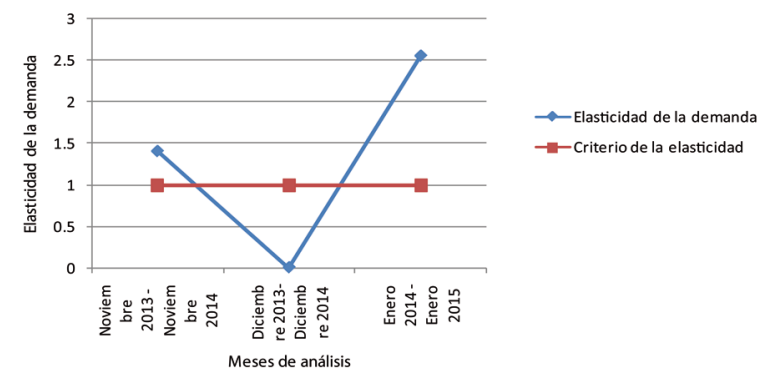

Figura 4. Evolución de la elasticidad de la demanda entre meses de diferentes años

En el figura 4 se muestra cómo la comparación entre meses desde noviembre de 2013 a noviembre de 2014 la demanda tiene una relación elástica, es decir que por cada aumento del precio en 1.5 punto hubo una disminución en el número de créditos. Para los siguientes meses donde el impuesto a SOLCA ya aparece en el listado de rubros cobrados en los estados de cuenta de los consumidores, la demanda en el producto financiero no mostró un aumento o disminución en los números de créditos solicitados a pesar que el costo del dinero se incrementó, dando como resultado una elasticidad nula.

Para el comparativo del mes de enero los consumidores tuvieron un comportamiento diferenciado a los meses anteriores ya que la relación precio con cantidad es directamente proporcional y sensible a cada cambio que el costo del dinero en los bancos se adicione. La aplicación del impuesto 
a cada rubro solicitado por concepto de préstamo de consumo cambio por completo el uso de esta herramienta de financiamiento y generando que los ecuatorianos sean más cautelosas en utilizar estos productos financieros.

CONCLUSIONES

En el presente artículo se realizó un análisis a través del criterio de la elasticidad de la demanda a fin de conocer si el aumento en el costo del dinero por medio de préstamos de consumo variaría en los meses de noviembre, diciembre y enero de 2015 por la inclusión del Impuesto a SOLCA que graba un $0.5 \%$ sobre cada dólar desembolsado por bancos del sector privado.

En el mes de noviembre los consumidores no mostraron una restricción en usar los préstamos de consumo para solventar sus necesidades diarias, quizás por desconocimiento o desinterés que el mismo afectaría a su cupo de crédito otorgado por los bancos privados. En el mes de diciembre, a pesar de las ofertas por

\section{REFERENCIAS BIBLIOGRÁFICAS}

Banco Central del Ecuador. (31 de diciembre de 2013). Banco Central del Ecuador. Recuperado el 08 de enero de 2015, de http://www.portal. bce.fin.ec/vto_bueno/seguridad/ComercioExteriorEst.jsp

BCE. (2014). Evolución del crédito del Sistema Financiero Privado. Banco Central del Ecuador, 1.

BCE. (2015). Evolución del crédito del Sistema Financiero Privado. Banco Central del Ecuador, 1.

Bigne, S., \& Cuenca, A. (2010). Investigación de mercados. Barcelona: MCGRAW- HILL.

Brown, L. O. (2009). Comercialización y análisis del mercado. Buenos Aires: Selcon.

Calderón, J. (2002). Comparación de los dos últimos años en los índices de políticas de ahorro y consumo en las familias del Ecuador. Revista académica de economía con el Número Internacional Normalizado de Publicaciones Seriadas ISSN 1696-8352, 2014.

Cervantes, J. G. (2002). Evaluación Económica Financiera. México D.F.: Universidad Nacional Autónoma de México.

Douglas, E., \& Finnerty, J. (2002). Fundamentos de admnistración financiera. México: Pearson.

El Universo. (29 de julio de 2014). Código revive impuesto de $0,5 \%$ para créditos para beneficiar a Solca. El Universo, pág. 1.

Fernández, S. (2007). Los proyectos de inversión: evaluación financiera. Costa Rica: Tecnológica de Costa Rica. las festividades navideñas se tenía previsto que aumenten los niveles de consumo, sin embargo con relación al mes anterior mostró cambio en la demanda realizada, ya que los consumidores en este mes prefirieron no usar esta herramienta financiera como medio de pago por el costo que soportaría a través del nuevo impuesto propuesto.

En el mes de diciembre los bancos privados no pudieron aumentar su participación de mercado, ni tampoco perdieron parte del mismo. En cambio, en el mes de enero el comportamiento de este segmento de mercado es totalmente elástica, donde la demanda tiende a disminuir su consumo por la ratificación de pagar adicionalmente un $0.5 \%$ por cada dólar consumido, llegando a concluir que la implementación de este rubro si afecta en los hábitos de consumo de los ecuatorianos y que actualmente cuidan su bolsillo antes d utilizar esta forma de financiamiento.

Gallardo, J. (2002). Evaluación económica y financiera. México D.F.: Universidad Nacional Autónoma de México.

González, R., \& Rodríguez, F. (2006). "Análisis de la percepción del consumidor sobre los servicios financieros de la Banca Consumo y definición de los diversos perfiles demográficos y de su conducta de compra". Guayaquil: Espol.

Hitt, M. (2002). Administración. Madrid: Pearson. Huerta, J. (2010). Dinero, crédito bancario y ciclos. Revista de Economía Aplicada E Número 18 (vol. VI), 1998, págs. 175 a 182.

Hurtado, J. (2000). Metodología de la investigación holítica. Caracas: Fundacion Sypal 1era. Edición.

Larraín, F., \& Sachs, J. (2010). Macroeconomía en la economía global. Mexico: Limusa.

Lawrence, G. (2010). Principios de administración financiera. México D.F.: Pearson Education.

Luchena, G. (2007). Retenciones e ingresos a cuenta en el impuesto sobre rentas. Madrid: Universidad de Castilla.

Morales, J. (14 de abril de 2015). Incidencia del Impuesto a Solca sobre los préstamos de consumo. (M. Pelchor, Entrevistador)

MSP. (04 de febrero de 2013). Ministerio de Salud Pública. Recuperado el 27 de diciembre de 2014, de http://instituciones.msp.gob.ec/ somossalud/index.php/enterate/200-el-30-a- 
40-de-casos-de-cancer-podrian-evitarse-si-secambian-cinco-malos-habitos.

Navarro, D. (2003). Administración financiera. Manizales: Universidad Nacional de Colombia.

Parkin, M. (2007). Macroeconomía: versión para latinoamerica. México: Pearson Educación.

Phillip, P. (2011). Economia de Empresa. México: Pearson Educación.

Plus, I. (6 de Septiembre de 2007). Instrumentos Financieros: Reconocimiento y Medición. Recuperado el 7 de octubre de 2014, de Instrumentos Financieros: Reconocimiento y Medición.
SOLCA. (01 de agosto de 2011). Sociedad de Lucha Contra el Cáncer del Ecuador. Recuperado el 04 de noviembre de 2014, de http://www.solca. med.ec/

Superintendencia de Bancos y Seguros del Ecuador. (25 de abril de 2015). SBS. Obtenido de http://portaldelusuario.sbs.gob.ec/contenido. php?id_contenido=23. 